\title{
3 Research Square

\section{UBE2T is upregulated, predicts poor prognosis, and promotes cell proliferation and invasion via inhibiting autophagy in ovarian cancer}

Hongyan Huang

Central South University

Wei Huang

Central South University

Yuzhen Xiao

Central South University

Lei Wang

Central South University

Tingting Zhang

Central South University

Xiaoling Fang

Central South University

Xiaomeng Xia ( $\nabla$ xiaxiaomeng@csu.edu.cn )

the SecondXiangya Hospital Central South University

Research

Keywords: Ovarian cancer, UBE2T, malignant progression, AKT/mTOR, Autophagy

Posted Date: July 24th, 2020

DOI: https://doi.org/10.21203/rs.3.rs-45356/v1

License: (c) (i) This work is licensed under a Creative Commons Attribution 4.0 International License.

Read Full License 


\section{Abstract}

Background: Aberrant upregulation and oncogenic roles of UBE2T have been revealed in several cancers. However, the expression, clinical significance, and functions of UBE2T has not been explored in ovarian cancer (OC).

Methods: In this study, the mRNA and protein expression of UBE2T in OC were detected via analyzing the online databases and immunohistochemical staining. Moreover, relations of UBE2T expression with clincopathological features and prognosis $\mathrm{OC}$ patients were further analyzed. Besides, the effects of UBE2T knockdown on growth, proliferation, and invasion of OC cells were investigated by CCK-8, plate clone formation, and Transwell assays. Finally, the underlying mechanism of UBE2T associated functions in $\mathrm{OC}$ was analyzed.

Results: The results indicated that UBE2T was significantly upregulated in OC tissues. UBE2T expression was notably correlated with clinical features such as primary T stage, TNM stage in OC patients. UBE2T, acting as an independent prognostic indicator, was inversely associated with prognosis of OC patients. UBE2T knockdown remarkably suppressed the growth, proliferation, and invasion of OC cells indicating by impaired cell viability, less cell clones and invasive cells. Mechanistically, the oncogenic roles of UBE2T was exerted by inhibiting autophagy via maintaining AKT/mTOR activity in OC.

Conclusion: Collectively, our findings confirm UBE2T upregulation predicts poor prognosis and promotes malignant progression via suppressing autophagy in $\mathrm{OC}$, suggesting the promising values of UBE2T both in diagnosis and treatment of OC.

\section{Introduction}

Ovarian cancer (OC) is one of aggressive gynecologic cancers with the poor prognosis, whose five-year overall survival rate is less than $50 \%$. Consistent with the circumstances for most carcinomas, the favorable therapeutic and prognostic outcomes are just achievable for OC patients at early stage. However, the actual rate of OC patients diagnosed at early stage is as low as $20 \%$, indicating the most OC patients are diagnosed at advanced stages, which largely accounts for the unsatisfactory prognosis of OC patients [1, 2]. Obviously, the unfavorable outcomes suggest the defects of the current biomarkers and treatments of $\mathrm{OC}$ and it is vital to explore novel diagnostic and prognostic biomarkers, and therapeutic targets to improve the efficiency of diagnosis and treatment in OC.

Dysregulations of catalytic enzymes, involving regulation of protein stability and degradation, exert critical roles in malignant transformation and progression of cancers $[3,4]$. Severing as the main pathway of protein degradation, ubiquitin-proteasome system (UPS) is closely related with carcinogenesis, reflected by the aberrant expression of proteins accounting for catalyzing the ubiquitination reaction [3, 5]. The substrates ubiquitination are sequentially catalyzed by ubiquitin-activating enzymes (E1), ubiquitin-conjugating enzymes (E2), and ubiquitin ligases (E3), with ubiquitin. Given their capacities of determining substrate specificity in ubiquitination, the expressions and roles of E3 ligases have been 
widely explored in cancers and their roles in malignances are comprehensively revealed $[5,6]$. Interestingly, apart from E3 ligases, the aberrant expressions and critical functions of ubiquitinconjugating enzymes, being considered as a family of constitutive enzymes before, in tumorigenesis have been indicated by the recent work [7].

Ubiquitin-conjugating enzyme E2T (UBE2T) is firstly identified in the study of Fanconi anaemia syndrome (FA) and plays vital roles in the development of FA via catalyzing the mono-ubiquitination of FANCD2 and FANCl. Accordingly, high mutation rate of UBE2T is observed in patients with FA [8]. Recently, the roles of UBE2T have been revealed in cancers. Upregulation of UBE2T, severing as an unfavorable prognostic indicator, has been confirmed in both solid tumors, such as gastric cancer $[9,10]$, hepatocellular carcinoma [11, 12], breast cancer [13], lung cancer [13, 14], nasopharyngeal carcinoma [15], osteosarcoma[16], prostate cancer[17] and renal cell carcinoma[18], and non-solid tumor like multiple myeloma (MM) [19]. Consistently, UBE2T depletion notably suppresses malignant progression via modulation the activity AKT [18] and p53 [12]. Nevertheless, the expression, clinical significance, functions and the corresponding mechanism of UBE2T has not been revealed in OC.

Therefore, in this study, we examined the mRNA and protein expression, prognostic value of UBE2T in OC via mining the online data and immunohistochemistry assay in local OC cohort. Moreover, we also explored the primary functions of UBE2T in OC by detecting the effects of UBE2T knockdown on cell phenotypes, including growth, proliferation, and invasion, and analyzed the role of autophagy in UBE2T related functions. The outcomes reveal that UBE2T mRNA and protein is upregulated and severs as an indicator for poor prognosis of OC patients. Furthermore, UBE2T knockdown significantly activated autophagy via suppressing AKT/mTOR, and subsequently inhibits growth, proliferation, and invasion of OC cells.

side) $<0.05$ was considered statistically significant.

\section{Results}

\section{UBE2T is upregulated in OC.}

Firstly, we analyzed the expression of UBE2T via several web portals. As shown in Fig. 1A, compared with the normal counterparts, the mRNA expression of UBE2T was significantly upregulated in OC tissues reflected by two data sets in Oncomine [23]. Accordingly, through analyzing the data in GEPIA [24], which integrated the data from TCGA and GTEx databases, upregulation of UBE2T mRNA in OC was further validated (Fig. 1B). Furthermore, Mass-spectrometry-based proteomic data from UALCAN [25] indicated upregulation of UBE2T protein in OC (Fig. 1E). Finally, we analyzed the protein level of UBE2T in a cohort of $\mathrm{OC}$ via IHC staining. The results showed that the staining intensity of UBE2T was significantly stronger in OC specimens than in normal samples (Fig. 1F). Thus, these results revealed UBE2T was upregulated in OC both at mRNA and protein level.

\section{UBE2T correlates to the clinical features of patients with OC.}


Subsequently, we explored relation between UBE2T expression and clinopathological variables. According to Oncomine data, the UBE2T mRNA level was comparative among different histological types including mucinous, serous and endometrioid type of OC (Fig. 1C), which was confirmed by the results of IHC (Fig. $1 F$, Table 1). Interestingly, based on the data from GEPIA, UBE2T mRNA level was inversely associated with the stage of OC patients (Fig. 1D). However, the IHC data revealed that although no significant difference of UBE2T protein among stage I, II, III and IV, notable upregulation of UBE2T was observed in advance stage(III+IV) OC tissues than that in early stage (I+II) (Fig. 1F, Table 1). Moreover, UBE2T level positively correlated with primary T stages in OC (Table 1). However, possibly due to the limited sample volumes, UBE2T showed no significant relation with distant metastasis and lymph node metastasis (Table 1). These results revealed that UBE2T was associated with stages of OC patients indicating UBE2T may involve in the progression of $\mathrm{OC}$.

\section{UBE2T inversely associated with the prognostic outcomes and served as an independent indicator for OC patients.}

Next, we investigated the relation between UBE2T and prognosis in OC via online and experimental data. The data from Kaplan-Meier Plotter portal [26] indicated UBE2T upregulation was negatively correlated with overall survival (OS), progression-free survival (PFS), and post-progression survival (PPS) of OC patients (Fig. 2A). Besides, GEPIA data confirmed that higher UBE2T mRNA was significantly correlated poor OS of OC patients (Fig. 2B). Consistently, upregulation of UBE2T protein served as a poor indicator for $\mathrm{OC}$ patients as well (Fig. $2 \mathrm{C}$ ). The univariate Cox proportional hazards regression analysis revealed that primary T stage, TNM stage, distant metastasis, and the level of UBE2T were significantly related to the OS of OC patients (Table 2). The multivariate Cox proportional hazards regression analysis indicated that UBE2T upregulation severed as an independent predictor for poor OS for OC patients (Table 2). Thus, these outcomes confirmed UBE2T was inversely related to the prognosis and served as an independent indicator for poor prognosis for $\mathrm{OC}$ patients.

\section{UBE2T depletion inhibits growth, proliferation, and invasion of OC cells.}

Next, we further explored the functions of UBE2T in OC. Therefore, we checked the expression of UBE2T, depleted UBE2T expressions with si-UBE2T transfection, and subsequently detected the influences on OC cells. As the qPCR and western blot results shown (Fig. 3A), both mRNA and protein expression of UBE2T were remarkably higher in OC cells, OVCAR3 and SKOV3 than that in IOSE80 cell, an immortalized normal ovarian epithelial cell line. As indicated by Fig. 3B, UBE2T expression was successfully knocked down in OVCAR3 and SKOV3 cells. The CCK-8, plate clone formation and Transwell invasion assays manifested UBE2T depletion significantly suppressed the growth, proliferation, and invasion of OC cells, demonstrated by impaired viability (Fig. 3C), less cell clones (Fig. 3D) and invasive cells (Fig. 3E). Herein, these results unveiled UBE2T depletion could inhibit growth, proliferation and invasion of OC cells.

UBE2T knockdown suppresses malignant progression of OC cells via activating autophagy by inhibiting AKT/mTOR. 
The roles of autophagy in $\mathrm{OC}$ have been widely explored [27, 28]. Considering the regulatory effects of UBE2T on AKT [18] and p53 [12], which are vital regulators of autophagy [28], we explored the function of UBE2T in autophagy of OC cells. As the results shown, UBE2T knockdown activated the autophagy of OC cells indicating by upregulated BECN1, accumulated LC3B, and decreased p62 (Fig. 4A). Moreover, the accumulative effects of LC3B were augmented under CQ $(50 \mu \mathrm{M})$ treatment (Fig. 4B), further confirming autophagy activation induced by UBE2T knockdown. Simultaneously, UBE2T depletion inhibited AKT/mTOR, reflected by decreased p-AKT (S473) and p-mTOR (S2448) (Fig. 4A), but exerted no influence on p53 (Fig. 4A), suggesting AKT/mTOR axis mediating the regulation of UBE2T on autophagy. Therefore, further experiments were performed to validate this notion. Accordingly, ectopic UBE2T expression could re-inhibit autophagy in OC cells with endogenous UBE2T knockdown, and the rescue

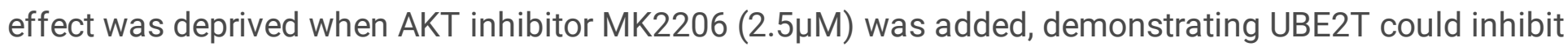
autophagy via sustaining AKT/mTOR activity (Fig. 4C). Meanwhile, consistent phenotypes were observed indicating by CCK-8 (Fig. 4D), plate clone formation (Fig. 4E), and Transwell invasion results (Fig. 4F). Thus, we revealed that UBE2T knockdown suppressed malignant progression of OC cells via activating autophagy by inhibiting AKT/mTOR.

\section{Discussion}

Here, we demonstrated that the mRNA and protein level of UBE2T was notably upregulated in OC tissues and cells. Upregulation of UBE2T was inversely correlated with stages and prognosis of OC patients. UBE2T inhibition remarkably suppressed the growth, proliferation and invasion of OC cells via activating autophagy by suppressing AKT/mTOR. Collectively, our findings suggest the promising values of UBE2T as a prognostic biomarker and therapeutic target in $\mathrm{OC}$.

The roles of UBE2T have been primarily revealed in FA related ubiquitin signaling [8]. UBE2T is critical for maintenance of genome integrity in FA involving its regulatory roles in mono-ubiquitination of FANCD2 and FANCI. Notably, FA patients bearing related gene mutations are particularly prone to malignant outcomes, suggesting the vital roles of FA related genes in tumorigenesis [8]. Indeed, aberrant upregulation of UBE2T has been observed in a host of cancers. For example, UBE2T is remarkably upregulated in MM cells, especially in the early stage. UBE2T level is significantly associated with IgG serotype of MM and UBE2T upregulation predicts poor prognosis, including OS and event-free survival time, of patients with MM [29]. Furthermore, UBE2T is upregulated in gastric cancer tissues and cells, positively associated with poor differentiation, advanced $T$ stage, and short OS time in patients with gastric cancer $[9,10]$. Accordingly, UBE2T upregulation, severing as a poor prognostic biomarker, has been confirmed in nasopharyngeal carcinoma [20], osteosarcoma [16], lung cancer [13, 14], breast cancer [16], prostate and hepatocellular cancer $[11,12,17,26]$. Consistently, we demonstrated that UBE2T mRNA and protein was upregulated in OC tissues and cells and UBE2T upregulation was associated with stages and poor prognosis $\mathrm{OC}$ patients.

The functions of UBE2T have been explored in cancers. UBE2T knockdown exerts significantly inhibitory effects on cell characteristics via different mechanisms. By inhibiting AKT and related pathways, UBE2T 
depletion remarkably suppresses cell proliferation, migration, and invasion of osteosarcoma [30], nasopharyngeal carcinoma [15], liver cancer [12], and renal cell carcinoma [18]. Besides, the suppressive roles of UBE2T knockdown on bladder cancer and hepatocellular carcinoma are mainly caused by apoptosis [31] and cell cycle arrest [11]. Furthermore, the oncogenic roles of UBE2T closely depend on their enzymatic activity. UBE2T can inactivate p53 and BRAC1, promote proliferation of hepatocellular carcinoma and breast cancer cells via directly catalyzing their mono-ubiquitination [11, 32]. Consistently, UBE2T knockdown inhibited AKT/mTOR and exerted notably inhibitory effects on growth, proliferation, and invasion of $O C$ cells.

Ubiquitin conjugating enzymes can exert essential regulatory roles in physiopathologic processes, including cancers, via modulating autophagy $[8,33,34]$. UBE2L6 depletion can activate autophagy and regulate the chemosensitivity of esophageal cancer cells [33]. Serving as either oncogenic or anti-tumor regulator, autophagy is widely implicated in regulation of stem maintenance, proliferation, invasion, metastasis, and therapy resistance of OC $[27,28,35]$. Here, we validated that UBE2T inhibition could promote autophagy, subsequently suppress the malignant characteristics of $\mathrm{OC}$ cells via constraining $\mathrm{AKT} / \mathrm{mTOR}$.

\section{Conclusion}

In conclusion, we initially and comprehensively explored the expression, clinical significance, fundamental functions, and underlying mechanisms of UBE2T in OC. The outcomes reveal that UBE2T is notably increased in OC, which severs as an indicator for poor prognosis in OC patients. UBE2T knockdown suppresses AKT/mTOR activity, subsequently activates autophagy, and eventually inhibits growth, proliferation, and invasion of $\mathrm{OC}$ cells. These findings contribute a better understanding of development and progression of $\mathrm{OC}$ and present UBE2T as a promising biomarker and therapeutic target in diagnosis and treatment of $\mathrm{OC}$, respectively.

\section{Methods}

\section{Tissue samples, Ethical Statement and Immunohistochemistry (IHC)}

An OC tissue chip, containing 70 OC tissues and 10 normal tissues (\#OVC1504) was purchased from the shanghai Superbiotek Pharmaceutical Technology Inc. (Shanghai, China). The study was approved by the Academic Ethics Committee of the Second Xiangya Hospital of Central South University and performed under the instructions of Declaration of Helsinki. IHC and scoring were applied according to our previous description [20] with primary antibody UBE2T (dilution: 1:50, BBI, Shanghai, China).

\section{Cell Culture}

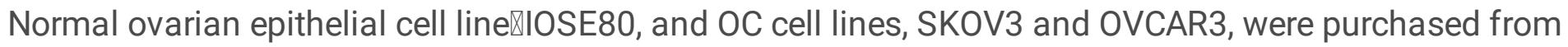
the American Type Culture Collection (ATCC, VA, USA). SKOV3 was maintained in RPMI-1640 medium plus $10 \%$ fetal bovine serum (FBS, Thermo, MA, USA). OVCAR3 and IOSE80 were cultured in DMEM 
medium plus $10 \%$ FBS (Thermo, MA, USA). The cells were culture in a humidified incubator at $37^{\circ} \mathrm{C}$ and $5 \% \mathrm{CO}_{2}$. The chloroquine (CQ, Sigma-Aldrich, MO, USA) and MK2206 (Selleck, TX, USA) were added into the culture medium for functional experiments as indicated in figure legends.

\section{Small interfering RNAs (siRNAs) and plasmids transfection}

The UBE2T siRNA (si-UBE2T) and scrambled siRNA (si-NC) were purchased from RiboBio Inc.

(Guangzhou, China). The UBE2T expression plasmid, pENTER-UBE2T, and the control plasmid, pENTERvector, were obtained from Vigene Inc. (Jinan, China). The si-UBE2T sequences are 5'-

GTCCTGGTTCATCTTAGTTAA-3', which targets the 3' untranslated region of UBE2T mRNA. The siNC sequences were not offered by the manufacturer. SKOV3 and OVCAR3 cells were transfected with siUBE2T or co-transfected with siUBE2T and pENTER-UBE2T using Lipofectamine ${ }^{\mathrm{TM}} 2000$ (Thermo, MA, USA) according to our previous description [21].

\section{RNA isolation and quantitative real-time PCR(qPCR)}

The total RNA isolation and qPCR was carried out as previous description [22]. The expressions of UBE2T was determined by qPCR with glyceraldehyde-3-phosphate dehydrogenase (GAPDH) as an endogenous control. The primer sequences of UBE2T and GAPDH are as follows. UBE2T, Forward 5'-

ATCCCTCAACATCGCAACTGT-3', Reverse 5'-CAGCCTCTGGTAGATTATCAAGC-3'; GAPDH, Forward 5'ATGGAGAAGGCTGGGGCTC-3', Reverse 5'-AAGTTGTCATGGATGACCTTG-3'.

\section{Cell growth assay}

The cell growth assay was performed as previously described [21]. The effects of UBE2T rescue and MK2206 addition on cell growth were reflected by inhibitory growth rate with si-NC group as control. The experiments were independently performed in triplicate.

\section{Plate clone formation assay}

Plate clone formation assay was carried out as previously described [22]. The experiments were independently performed for three times.

\section{Transwell invasion assay}

Transwell invasion assay was performed as previous description [22]. The experiments were independently performed for three times.

\section{Western blot}

The western blot was performed as previous description [22]. Primary antibodies, including UBE2T (BBI, Shanghai, China), p53(Santa Cruz, TX, USA), BECN1(Abclonal, Wuhan, China), p62(Abclonal, Wuhan, China), LC3A/B (Abclonal, Wuhan, China), p-AKT(S473) (CST, MA, USA), AKT(CST, MA, USA), mTOR (CST, 
MA, USA), p-mTOR(S2448) (CST, MA, USA), $\beta$-actin (Proteintech, Wuhan, China), were used to detect their levels under specific treatments.

\section{Statistical analysis}

Statistical analyses and statistical charts were analyzed and produced using SPSS20.0 software and GraphPad Prism version 8. For comparisons between two groups, a Student t-test or chi-square test was carried out. Survival curves were obtained via Kaplan-Meier method, and the statistical analysis was evaluated by Log-rank test. The univariate and multivariate Cox regression was performed to analyze the relationship of among UBE2T expression, clinicopathological parameters, and survival in OC patients. For all analyses, $P$ (two side) $<0.05$ was considered statistically significant.

\section{Declarations}

\section{Ethics approval and consent to participate}

The study was approved by the Academic Ethics Committee of the Second Xiangya Hospital of Central South University (OV_XXM_20191128) and performed under the instructions of Declaration of Helsinki. The informed consent were obtained from the all enrolled patients.

\section{Consent for publication}

Not applicable.

\section{Availability of data and materials}

The online databases, including Oncomine(www.oncomine.org), GEPIA(gepia.cancer-pku.cn), and UALCAN(ualcan.path.uab.edu), were used. Other data and materials are available from the corresponding author on reasonable request.

\section{Funding}

The present study was granted by the National Natural Science Foundation of China (nos. 81702924, 81671437, 81801425, and 81771558) and the Natural Science Foundation of Hunan Province of China (nos. 2018JJ3811).

\section{Author's contributions}

X-M. X and WH designed this study, wrote and revised the manuscript. X-M. X and X-L. F supervised this study. $\mathrm{H}-\mathrm{Y}$. $\mathrm{H}$ and $\mathrm{WH}$ performed the most experiments. Y-Z. $\mathrm{X}$ and LW performed the online analyses. T-T. $Z$ and LW contributed to the statistical analysis and picture process.

\section{Acknowledgements}

Not applicable. 


\section{Competing interests}

There are no conflicts of interest in this work by all authors.

\section{References}

1. Nash Z, Menon U. Ovarian cancer screening: Current status and future directions. Best Pract Res Clin Obstet Gynaecol. 2020; 65:32-45.

2. Chandra A, Pius C, Nabeel M, Nair M, Vishwanatha JK, Ahmad S, Basha R. Ovarian cancer: Current status and strategies for improving therapeutic outcomes. Cancer Med. 2019; 8(16):7018-7031.

3. Zhang X, Linder S, Bazzaro M. Drug Development Targeting the Ubiquitin-Proteasome System (UPS) for the Treatment of Human Cancers. Cancers (Basel). 2020; 12(4):902.

4. Dong Z, Cui H. The Autophagy-Lysosomal Pathways and Their Emerging Roles in Modulating Proteostasis in Tumors. Cells. 2018; 8(1).

5. Yang H, Chen X, Li K, Cheaito H, Yang Q, Wu G, Liu J, Dou QP. Repurposing old drugs as new inhibitors of the ubiquitin-proteasome pathway for cancer treatment. Semin Cancer Biol. 2019; S1044-579X(19)30411-0.

6. Narayanan S, Cai CY, Assaraf YG, Guo HQ, Cui Q, Wei L, et al. Targeting the ubiquitin-proteasome pathway to overcome anti-cancer drug resistance. Drug Resist Updat. 2020; 48:100663.

7. Hosseini SM, Okoye I, Chaleshtari MG, Hazhirkarzar B, Mohamadnejad J, Azizi G, et al. E2 ubiquitinconjugating enzymes in cancer: Implications for immunotherapeutic interventions. Clin Chim Acta. 2019; 498:126-134.

8. Alpi AF, Chaugule V, Walden $\mathrm{H}$. Mechanism and disease association of E2-conjugating enzymes: lessons from UBE2T and UBE2L3. Biochem J. 2016; 473(20):3401-3419.

9. Luo C, Yao Y, Yu Z, Zhou H, Guo L, Zhang J, et al. UBE2T knockdown inhibits gastric cancer progression. Oncotarget. 2017; 8(20):32639-32654.

10. Yu H, Xiang P, Pan Q, Huang Y, Xie N, Zhu W. Ubiquitin-Conjugating Enzyme E2T is an Independent Prognostic Factor and Promotes Gastric Cancer Progression. Tumour Biol. 2016; 37(9):11723-11732.

11. Liu LL, Zhu JM, Yu XN, Zhu HR, Shi X, Bilegsaikhan E, et al. UBE2T promotes proliferation via G2/M checkpoint in hepatocellular carcinoma. Cancer Manag Res. 2019; 11:8359-8370.

12. Liu LP, Yang M, Peng QZ, Li MY, Zhang YS, Guo YH, et al. UBE2T promotes hepatocellular carcinoma cell growth via ubiquitination of p53. Biochem Biophys Res Commun. 2017; 493(1):20-27.

13. Perez-Pena J, Corrales-Sanchez V, Amir E, Pandiella A, Ocana A. Ubiquitin-conjugating enzyme E2T (UBE2T) and denticleless protein homolog (DTL) are linked to poor outcome in breast and lung cancers. Sci Rep. 2017; 7(1):17530.

14. Wu ZH, Zhang YJ, Sun HY. High ubiquitin conjugating enzyme E2 T mRNA expression and its prognostic significance in lung adenocarcinoma: A study based on the TCGA database. Medicine (Baltimore). 2020; 99(4):e18543. 
15. Hu W, Xiao L, Cao C, Hua S, Wu D. UBE2T promotes nasopharyngeal carcinoma cell proliferation, invasion, and metastasis by activating the AKT/GSK3beta/beta-catenin pathway. Oncotarget. 2016; 7(12):15161-15172.

16. Shen L, Zhao K, Li H, Ning B, Wang W, Liu R, Zhang Y, Zhang A. Downregulation of UBE2T can enhance the radiosensitivity of osteosarcoma in vitro and in vivo. Epigenomics. 2019; 11(11):12831305.

17. Wen M, Kwon Y, Wang Y, Mao JH, Wei G. Elevated expression of UBE2T exhibits oncogenic properties in human prostate cancer. Oncotarget. 2015; 6(28):25226-25239.

18. Hao P, Kang B, Li Y, Hao W, Ma F. UBE2T promotes proliferation and regulates PI3K/Akt signaling in renal cell carcinoma. Mol Med Rep. 2019; 20(2):1212-1220.

19. Alagpulinsa DA, Kumar S, Talluri S, Nanjappa P, Buon L, Chakraborty C, et al. Amplification and overexpression of E2 ubiquitin conjugase UBE2T promotes homologous recombination in multiple myeloma. Blood Adv. 2019; 3(23):3968-3972.

20. Liu J, Huang W, Ren C, Wen Q, Liu W, Yang X, et al. Flotillin-2 promotes metastasis of nasopharyngeal carcinoma by activating NF-kappaB and PI3K/Akt3 signaling pathways. Sci Rep. 2015; 5:11614.

21. Huang W, Liu J, Hu S, Shi G, Yong Z, Li J, et al. miR-181a Upregulation Promotes Radioresistance of Nasopharyngeal Carcinoma by Targeting RKIP. OncoTargets and therapy. 2019; 12:10873-10884.

22. Huang W, Zeng C, Hu S, Wang L, Liu J. ATG3, a Target of miR-431-5p, Promotes Proliferation and Invasion of Colon Cancer via Promoting Autophagy. Cancer Manag Res. 2019; 11:10275-10285.

23. Rhodes DR, Yu J, Shanker K, Deshpande N, Varambally R, Ghosh D, et al. ONCOMINE: a cancer microarray database and integrated data-mining platform. Neoplasia. 2004; 6(1):1-6.

24. Tang Z, Li C, Kang B, Gao G, Li C, Zhang Z. GEPIA: a web server for cancer and normal gene expression profiling and interactive analyses. Nucleic Acids Res. 2017; 45(W1):W98-W102.

25. Chandrashekar DS, Bashel B, Balasubramanya SAH, Creighton CJ, Ponce-Rodriguez I, Chakravarthi B, et al. UALCAN: A Portal for Facilitating Tumor Subgroup Gene Expression and Survival Analyses. Neoplasia. 2017; 19(8):649-658.

26. Nagy A, Lanczky A, Menyhart O, Gyorffy B. Validation of miRNA prognostic power in hepatocellular carcinoma using expression data of independent datasets. Sci Rep. 2018; 8(1):9227.

27. Thuwajit C, Ferraresi A, Titone R, Thuwajit P, Isidoro C. The metabolic cross-talk between epithelial cancer cells and stromal fibroblasts in ovarian cancer progression: Autophagy plays a role. Med Res Rev. 2018; 38(4):1235-1254.

28. Zhan L, Zhang Y, Wang W, Song E, Fan Y, Li J, et al. Autophagy as an emerging therapy target for ovarian carcinoma. Oncotarget. 2016; 7(50):83476-83487.

29. Zhang W, Zhang Y, Yang Z, Liu X, Yang P, Wang J, et al. High expression of UBE2T predicts poor prognosis and survival in multiple myeloma. Cancer Gene Ther. 2019; 26(11-12):347-355.

30. Wang $Y$, Leng $H$, Chen $H$, Wang L, Jiang N, Huo X, et al. Knockdown of UBE2T Inhibits Osteosarcoma Cell Proliferation, Migration, and Invasion by Suppressing the PI3K/Akt Signaling Pathway. Oncol 
Res. 2016; 24(5):361-369.

31. Gong YQ, Peng D, Ning XH, Yang XY, Li XS, Zhou LQ, et al. UBE2T silencing suppresses proliferation and induces cell cycle arrest and apoptosis in bladder cancer cells. Oncol Lett. 2016; 12(6):44854492.

32. Ueki T, Park JH, Nishidate T, Kijima K, Hirata K, Nakamura Y, et al. Ubiquitination and downregulation of BRCA1 by ubiquitin-conjugating enzyme E2T overexpression in human breast cancer cells. Cancer Res. 2009; 69(22):8752-8760.

33. Falvey CM, O'Donovan TR, El-Mashed S, Nyhan MJ, O'Reilly S, McKenna SL. UBE2L6/UBCH8 and ISG15 attenuate autophagy in esophageal cancer cells. Oncotarget. 2017; 8(14):23479-23491.

34. Geisler S, Vollmer S, Golombek S, Kahle PJ. The ubiquitin-conjugating enzymes UBE2N, UBE2L3 and UBE2D2/3 are essential for Parkin-dependent mitophagy. J Cell Sci. 2014; 127(Pt 15):3280-3293.

35. Wang Q, Bu S, Xin D, Li B, Wang L, Lai D. Autophagy Is Indispensable for the Self-Renewal and Quiescence of Ovarian Cancer Spheroid Cells with Stem Cell-Like Properties. Oxid Med Cell Longev. $2018 ; 2018: 7010472$.

\section{Tables}

Table 1. Correlation between UBE2T expression and clinicopathological characteristics in Ovarian Cancer ( $\mathrm{n}=70, \chi 2$ test)

\begin{tabular}{|c|c|c|c|c|c|}
\hline \multirow[t]{2}{*}{ Variables } & \multirow[t]{2}{*}{$\mathrm{n}$} & \multicolumn{2}{|c|}{ Expression level } & \multirow[t]{2}{*}{$x^{2}$} & \multirow[t]{2}{*}{$P$} \\
\hline & & Low (1-3) & High (4-6) & & \\
\hline \multicolumn{6}{|l|}{ Age(years) } \\
\hline$\geq 50$ & 37 & 9 & 28 & 1.204 & 0.273 \\
\hline$<50$ & 33 & 12 & 21 & & \\
\hline \multicolumn{6}{|c|}{ Primary tumor ( $\mathrm{T}$ ) stage } \\
\hline $\mathrm{T} 1-2$ & 36 & 18 & 18 & 14.118 & 0.000172 \\
\hline T3-4 & 34 & 3 & 31 & & \\
\hline \multicolumn{6}{|c|}{ Lymph node(N) metastasis } \\
\hline No & 67 & 21 & 46 & - & 0.549 \\
\hline N1-3 & 3 & 0 & 3 & & \\
\hline \multicolumn{6}{|c|}{ Distant metastasis (M) } \\
\hline M0 & 65 & 21 & 44 & - & 0.313 \\
\hline M1 & 5 & 0 & 5 & & \\
\hline \multicolumn{6}{|c|}{ Clinical TNM stage } \\
\hline I-II & 35 & 18 & 17 & 15.306 & 0.000091 \\
\hline III-IV & 35 & 3 & 32 & & \\
\hline \multicolumn{6}{|c|}{ Pathological Classification } \\
\hline Endometrioid & 15 & 6 & 9 & 2.074 & 0.549 \\
\hline Mucinous & 7 & 3 & 4 & & \\
\hline Serous & 48 & 12 & 36 & & \\
\hline
\end{tabular}


Table 2. Univariate analysis and Multivariate analysis of prognostic factors for overall survival using Cox proportional hazards regression model $(\mathrm{N}=70)$

\begin{tabular}{lcccc}
\hline \multirow{2}{*}{ Variables } & \multicolumn{2}{c}{ Univariate analysis } & \multicolumn{2}{c}{ Multivariate analysis } \\
\cline { 2 - 5 } & HR(95\% CI) & $P$ & HR(95\% CI) & $P$ \\
\hline Age(years) & $1.292(0.642-$ & 0.473 & $1.062 \llbracket 0.521-$ & 0.868 \\
प50 vs $\geq 50$ & $2.602)$ & & $2.166 \square$ & \\
Primary tumor (T) stage & $4.393(2.026-$ & 0.0002 & $/$ & $/$ \\
T1-2 vs T3-4 & $9.524)$ & & & \\
Lymph node(N) & $4.547(0.530-$ & 0.167 & $4.729(0.532-$ & 0.163 \\
metastasis & $39.010)$ & & $42.062)$ & \\
Distant metastasis (M) & $8.480(2.698-$ & 0.0003 & $6.428(1.994-$ & 0.002 \\
& $26.655)$ & & $20.720)$ & \\
TNM stage & $4.353(2.007-$ & 0.0003 & $/$ & $/$ \\
& $9.442)$ & & & \\
Pathological & $1.423(0.898-$ & 0.133 & $1.272(0.8-$ & 0.308 \\
Classification & $2.255)$ & & $2.022)$ & \\
UBE2T & $5.992(1.823-$ & 0.003 & $4.986(1.476-$ & $0.01^{\mathrm{a}}$ \\
High vs Low & $19.696)$ & & $16.849)$ & \\
\hline
\end{tabular}

HR:hazard ratio; 95\%CI:95\% confidence interval;

\section{Figures}


$\mathbf{A}$

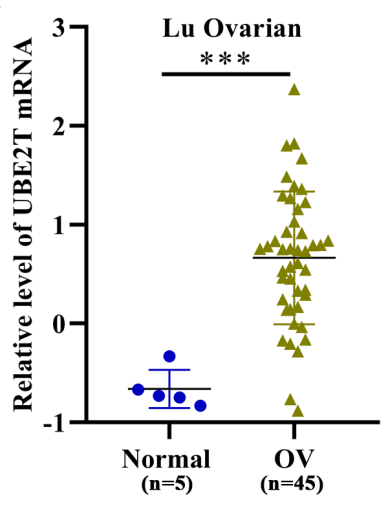

D

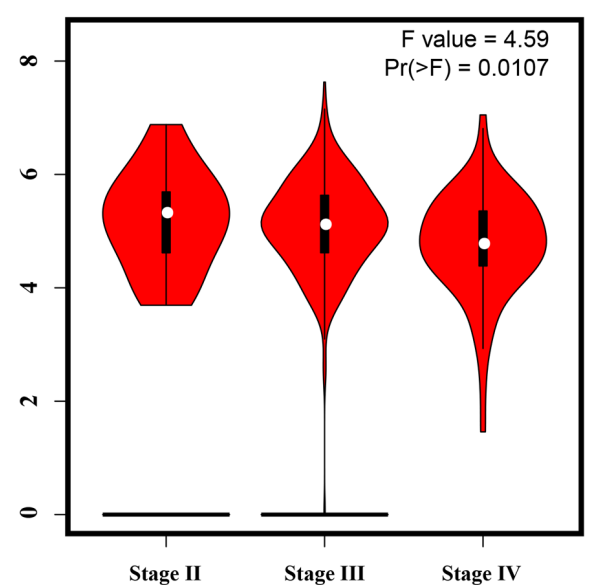

$\mathbf{E}$

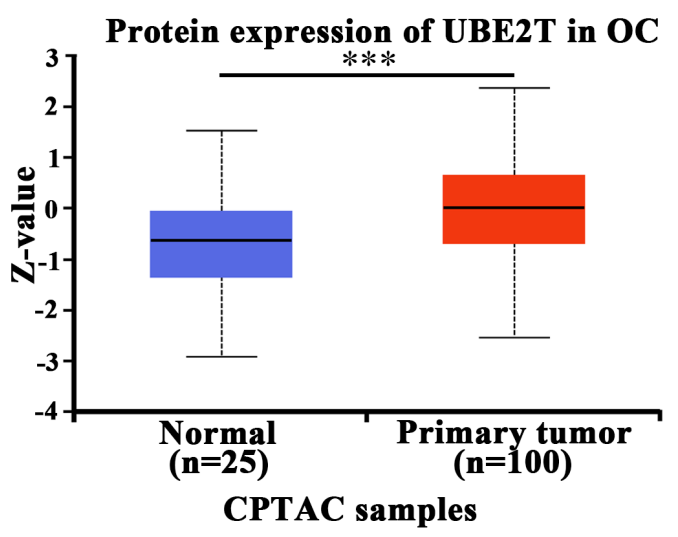

B

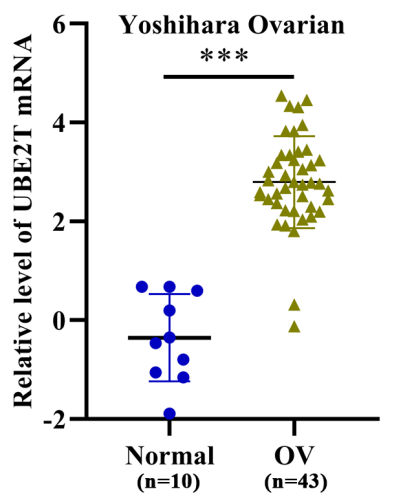

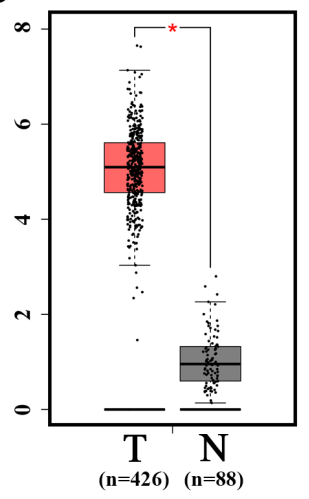

C

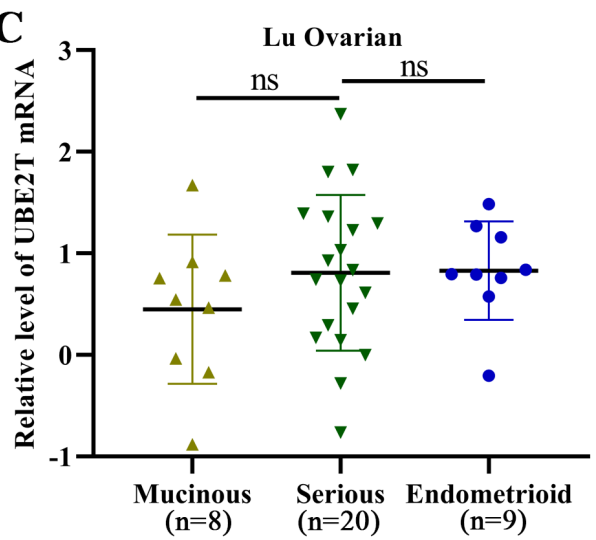

$\mathbf{F}$

F $\quad$ Endometrioid Mucinous
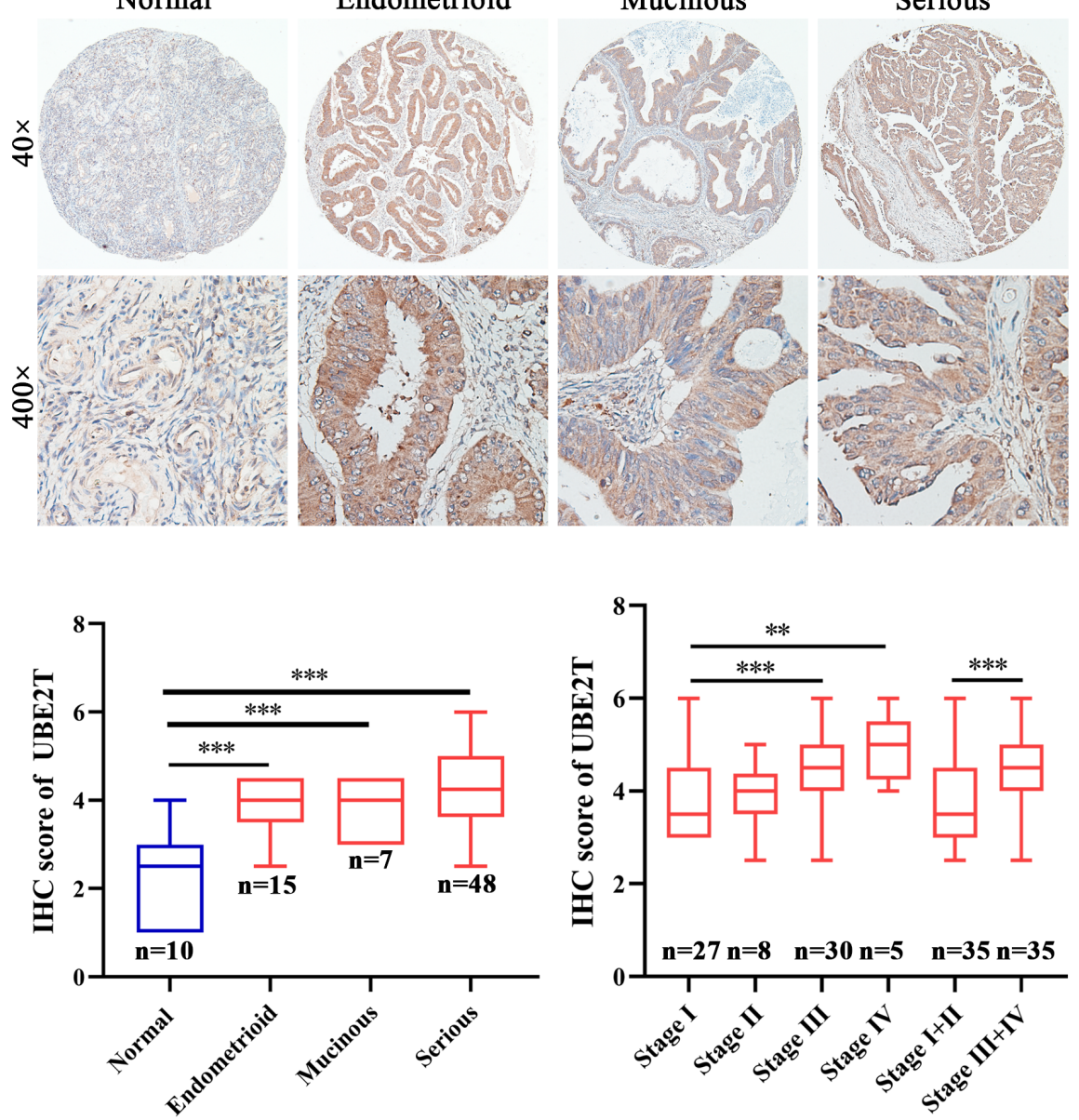

Figure 1

The expression of UBE2T in OC and its relation to clinical features of patients with OC. Oncomine data (A) and GEPIA data (B) indicated UBE2T mRNA upregulated in OC. (C) The Oncomine data showed no difference of UBE2T mRNA expression among mucinous, serious and endometriod OC tissues. (D) GEPIA data showed UBE2T mRNA significantly correlated to stage of OC. (F) UALCAN data showed UBE2T protein was upregulated in OC tissues. (F) IHC results of UBE2T protein level in normal tissues and OC tissues with different pathological types and stages. ${ }^{\star} P<0.05, * \star P<0.01$, ***P<0.001, ns, no significant statistical difference. 
A

UBE2T (223229_at)

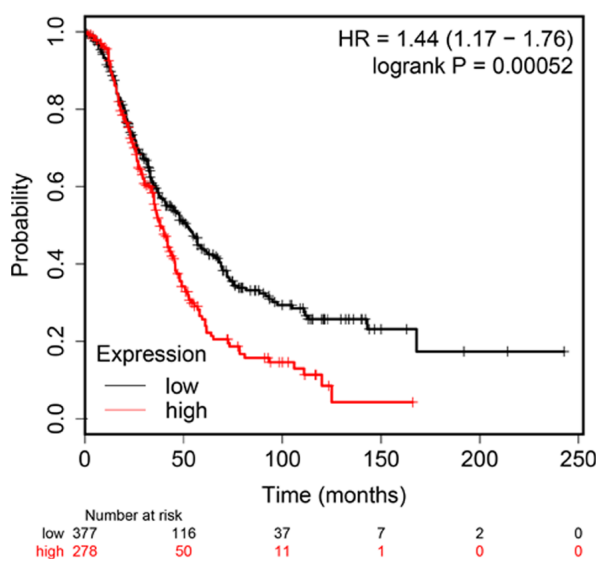

UBE2T (223229_at)

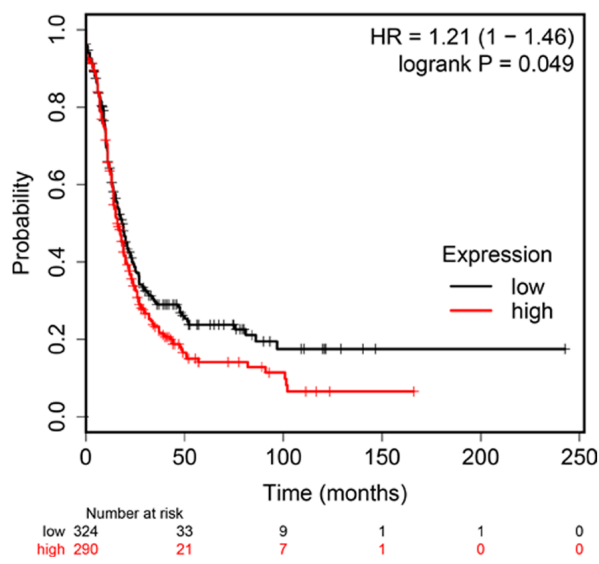

UBE2T (223229_at)

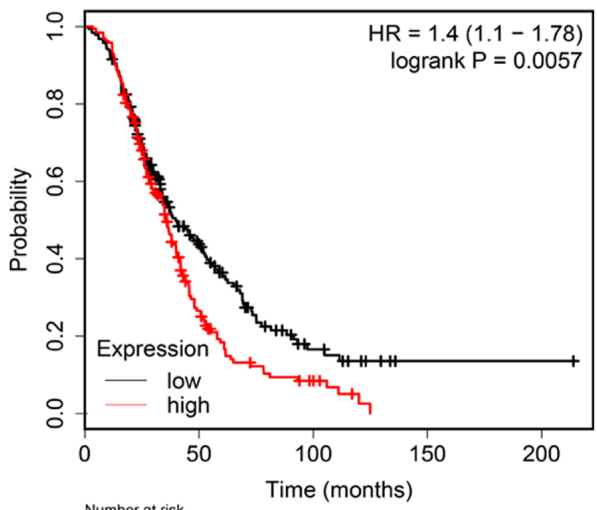

$\begin{array}{cc} & \text { Number at risk } \\ \text { low } 189 & 57 \\ \text { high } 193 & 35\end{array}$
B

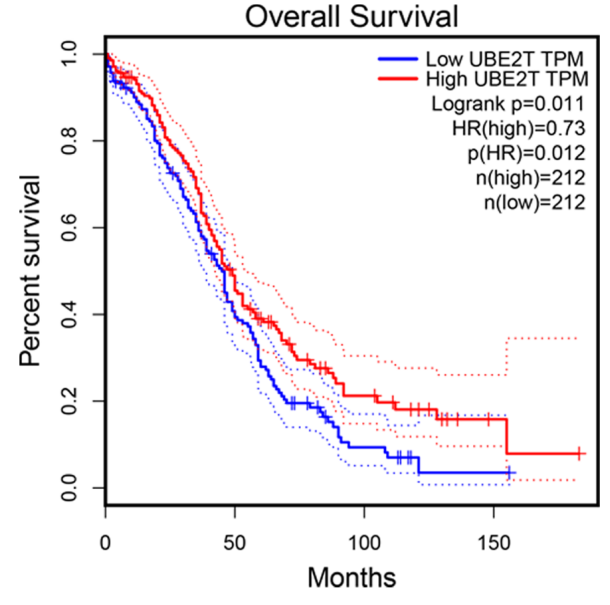

C

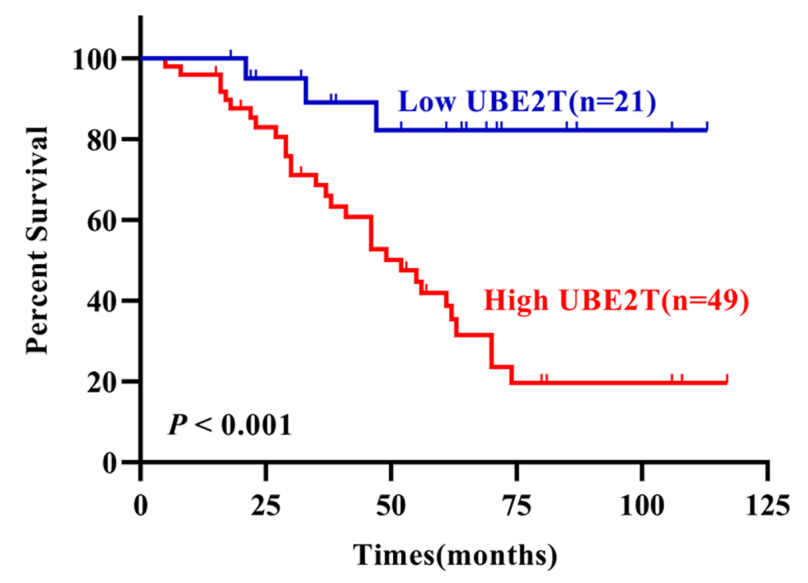

Figure 2

UBE2T upregulation predicts poor prognosis of patients with OC. (A) UBE2T mRNA level was inversely associated with OS (left panel), PFS (middle panel), and PPS (right panel) of OC patients indicated by Kaplan-Meier Plotter data. (B) UBE2T mRNA level was inversely associated with OS of OC patients indicated by GEPIA data. (C) UBE2T protein level was negatively related to OS of OC patients demonstrated by our data. 
$\mathbf{A}$

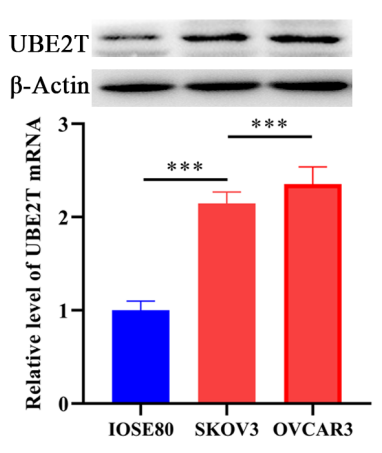

D

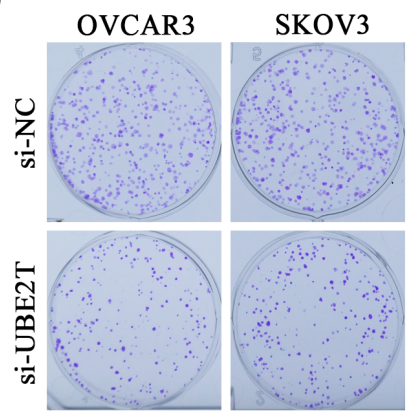

B

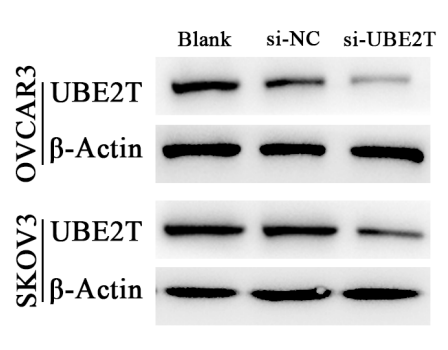

C

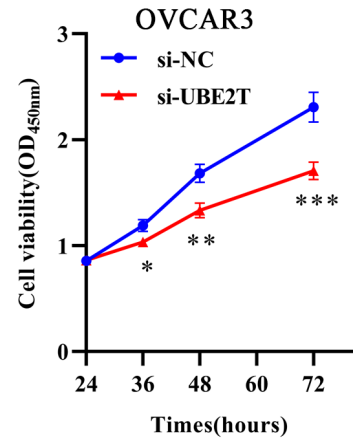

E

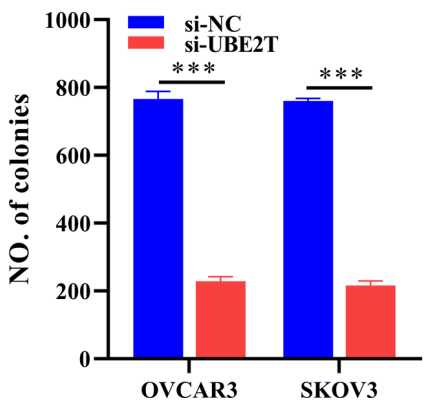

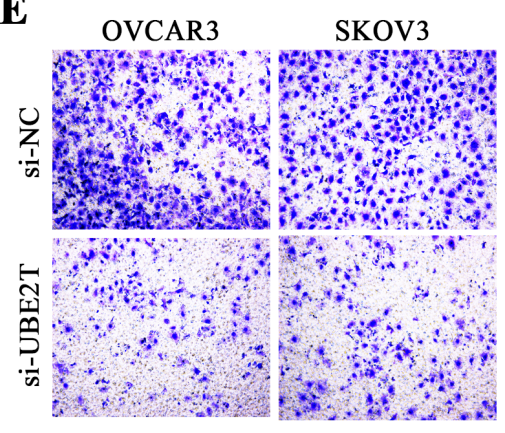

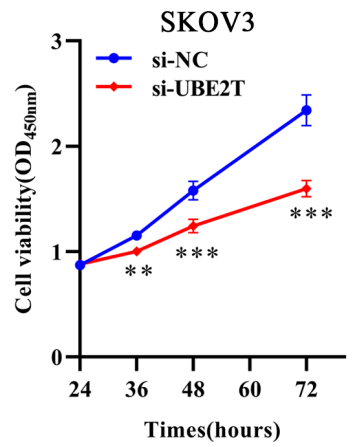

Times(hours)

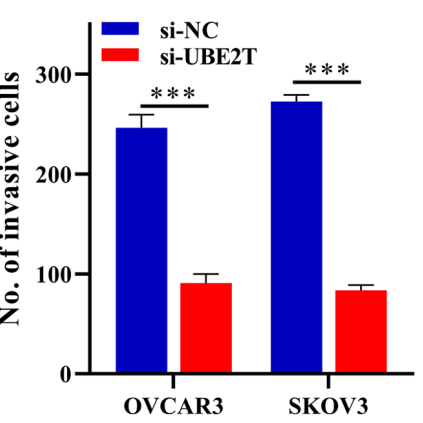

Figure 3

UBE2T depletion inhibits growth, proliferation and invasion of OC cells. (A) qPCR and western blot indicating the UBE2T expression among IOSE80, SKOV3 and OVCAR3 cell. (B) Western blot indicating the knockdown efficacy of siUBE2T in OC cells. Analyzing the effects of UBE2T knockdown on growth, proliferation, and invasion via CCK-8 (C), plate clone formation (D), and transwell invasion (E). ${ }^{*}<<0.05$, $\star \star P<0.01, * \star * P<0.001$. 

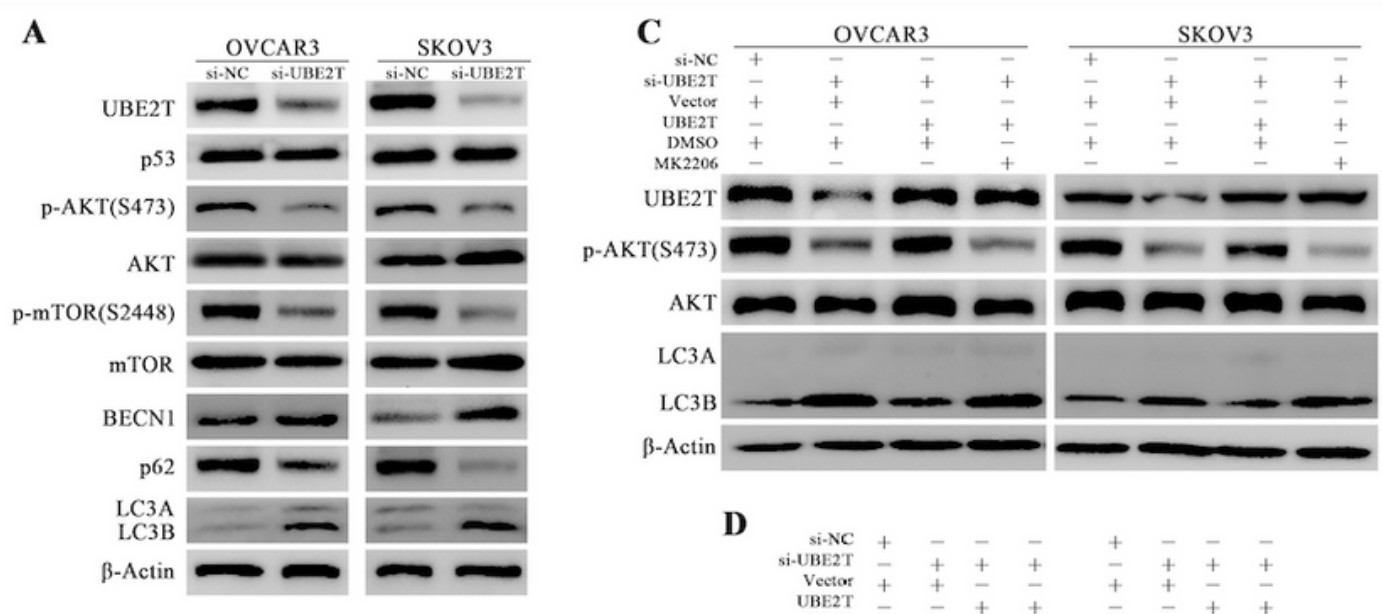

B

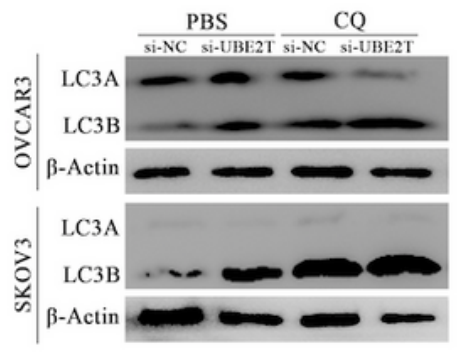

D

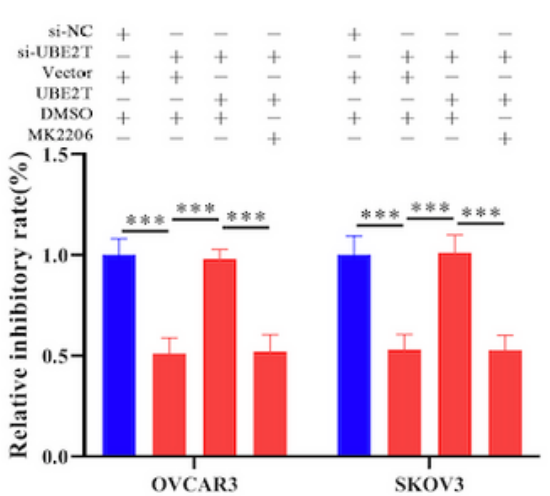

$\mathbf{E}$
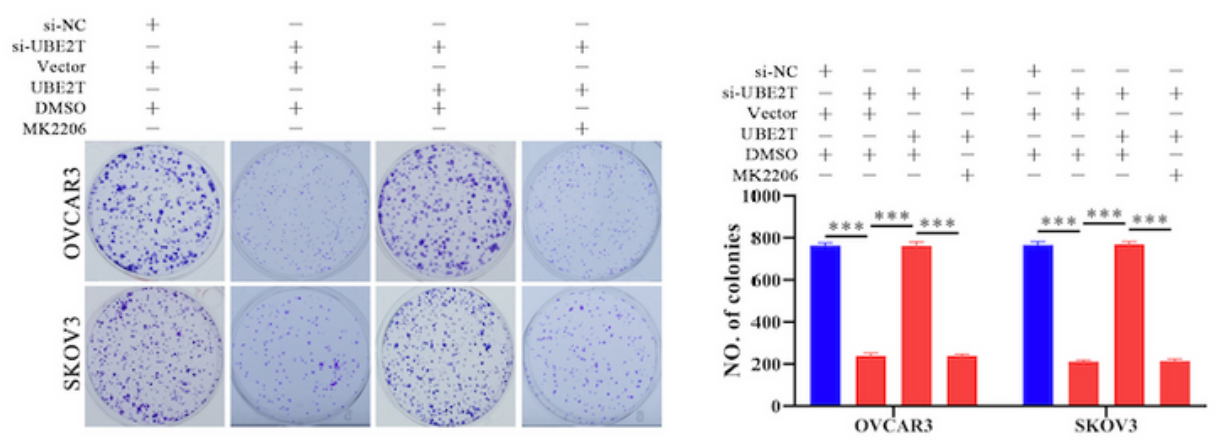

F
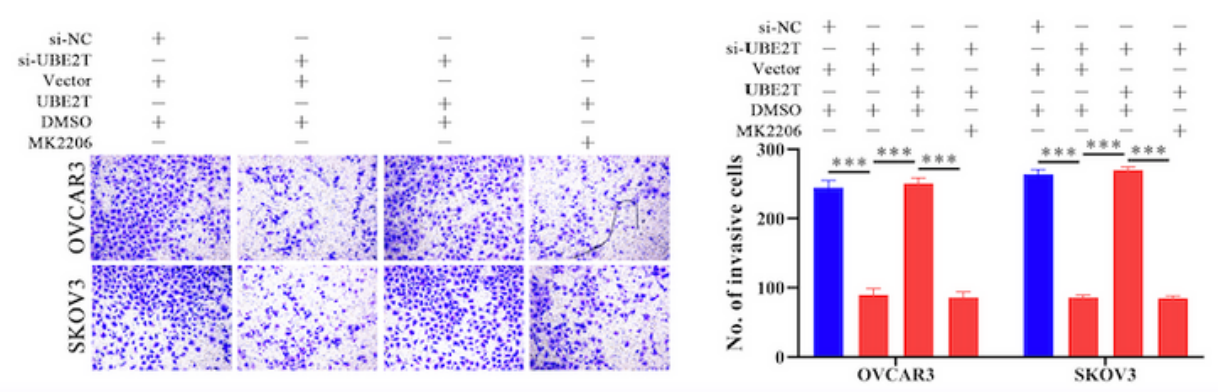

Figure 4

UBE2T depletion inhibited malignant progression of OC cells by inducing autophagy via suppressing AKT/mTOR axis. (A) Western blot indicating the level of p53, p-AKT (S473), p-mTOR (S2448), and autophagy related proteins in OC cells with UBE2T knockdown. (B) Western blot indicating the accumulated LC3B of OC cells with UBE2T knockdown under CQ $(50 \mu \mathrm{M})$ treatment. (C) Western blot indicating the influences of MK2206 $(2.5 \mu \mathrm{M})$ on the rescue effects of UBE2T on autophagy in OC cells 
with UBE2T knockdown. CCK-8 (D), plate clone formation (E), and transwell invasion (F) assays indicating the influences of MK2206 $(2.5 \mu \mathrm{M})$ on the rescue effects of UBE2T on autophagy in OC cells with UBE2T knockdown. 